BULLETIN OF THE

AMERICAN MATHEMATICAL SOCIETY

Volume 77, Number 6, November 1971

\title{
BESSEL POTENTIALS. INCLUSION RELATIONS AMONG CLASSES OF EXCEPTIONAL SETS
}

\author{
BY DAVID R. ADAMS AND NORMAN G. MEYERS
}

Communicated by James Serrin, June 17, 1971

1. Let $g_{\alpha}=g_{\alpha}(x)$ be the Bessel kernel of order $\alpha, 0<\alpha<+\infty$, on $R^{n} ; g_{\alpha}$ is the Fourier transform of $(2 \pi)^{-n / 2}\left(1+|\zeta|^{2}\right)^{-\alpha / 2}$. For $1<p<\infty$, we define a capacity $B_{\alpha, p}$ (referred to as Bessel capacity): for $A \subset R^{n}$,

$$
B(A)=B_{\alpha, p}(A)=\inf _{f} \int f(x)^{p} d x
$$

the infimum being taken over all functions $f$ in $L_{p}^{+}=L_{p}^{+}\left(R^{n}\right)$-positive functions in the Lebesgue class-such that $g_{\alpha} * f(x) \geqq 1$ for all $x \in A$. The capacities $B_{\alpha, p}$ have been studied extensively in [4]. It is an easy consequence of the definition of $B_{\alpha, p}$ that: $B_{\alpha, p}(A)=0$ if and only if there is an $f \in L_{p}^{+}$such that $g_{\alpha} * f(x)=+\infty$ on $A$.

Variants of the Bessel capacities occur for instance in [1], [3], [5].

Our purpose here is to announce results on the relations between the $B^{\prime}$ 's for various pairs $(\alpha, p)$. We say that the Bessel capacity $B$ is stronger than the Bessel capacity $B^{\prime}$ (written $B^{\prime} \preceq B$ ) if $B(A)=0$ always implies $B^{\prime}(A)=0$. If in addition, there is a set $A$ such that $B(A)>0$ but $B^{\prime}(A)=0$ we say $B$ is strictly stronger than $B^{\prime}\left(B^{\prime} \prec B\right)$. These are the relations between $B$ and $B^{\prime}$. If both $B^{\prime} \preceq B$ and $B \preceq B^{\prime}$ hold, we say $B$ is equivalent to $B^{\prime}\left(B \sim B^{\prime}\right)$. In addition to the relations among the $B$ 's, we also give some results concerning relations between Bessel capacities, Hausdorff measures, and classical capacities ( $C_{k}$ below). These classical capacities can be viewed as a special case of general $L_{p}$-capacities of [4] when $p=1$ or $p=2$.

Notation. wei $B=$ weight of $B_{\alpha, p}=\alpha p$; ord $B=$ order of $B_{\alpha, p}=\alpha$; ind $B=$ index of $B_{\alpha, p}=(\alpha, p)$. By $(\alpha, p) \prec(\beta, q)$ we shall mean either $\alpha p<\beta q$, or $\alpha p=\beta q$ and $\alpha<\beta$.

2. The principal result is

Theorem 1. If $B$ and $B^{\prime}$ are two Bessel capacities,

(i) $B^{\prime} \prec B$ if and only if ind $B^{\prime} \prec$ ind $B$ and wei $B^{\prime} \leqq n$.

(ii) $B^{\prime} \sim B$ if and only if ind $B^{\prime}=$ ind $B$ or wei $B$ and wei $B^{\prime}>n$.

AMS 1970 subject classifications. Primary 31C15, 31B15; Secondary 26A33. 
Let $k=k(r), r=|x|$, be a nonincreasing lower semicontinuous function on $[0,+\infty)$ (referred to as a kernel). Define the classical capacity

$$
C_{k}(K)=\sup _{\mu}\|\mu\|_{1},
$$

the supremum being over all positive Radon measures with finite total variation $\|\mu\|_{1}$ and whose supports are in the given compact set $K$. $C_{k}^{*}$ denotes the usual extension of $C_{k}$ to an outer capacity.

We are interested in $k(r)=g_{\alpha, q}(r) \equiv g_{\alpha}(r) \cdot l_{q}(r)$, where $l_{q}(r)$ $=(\log 1 / r)^{q-2}$ for $0<r \leqq r_{0}<1$ and constantly equal to $l_{q}\left(r_{0}\right)$ for $r>r_{0}$. For this choice of $k$ we denote $C_{k}^{*}$ by $B_{(\alpha, q)}$.

Theorem 2. (i) If $\alpha p=n$,

(a) $1<p<q<\infty$ implies $B_{(n, q)} \preceq B_{\alpha, p}$;

(b) $1<q<p<\infty$ implies $B_{\alpha, p} \preceq B_{(n, q)}$.

(ii) If $\alpha p<n$,

(a) $2 \leqq p<q<\infty$ implies $B_{(\alpha p, q)} \preceq B_{\alpha, p}$;

(b) $1<q<p \leqq 2$ implies $B_{\alpha, p} \preceq B_{(\alpha p, q)}$.

The relation $B_{(\alpha p, q)} \preceq B_{\alpha, p}$ for $\alpha p<n, 1<p<q<2$, is false in contrast to (i). An example showing this can easily be constructed using [6].

If $h=h(r)$ is nondecreasing with $h(0)=0$, the Hausdorff $h$-measure is defined as

$$
H_{h}(A)=\lim _{\boldsymbol{\epsilon} \rightarrow 0} \inf \sum_{i} h\left(r_{i}\right)
$$

the infimum being over countable covers of $A$ by balls of radius $r_{i} \leqq \epsilon, \epsilon>0$.

Theorem 3. (i) If $\alpha p=n$,

$$
\begin{aligned}
& B_{\alpha, p} \preceq H_{h}, \quad \text { for } h(r)=(\log 1 / r)^{1-p} \quad\left(0 \leqq r \leqq \frac{1}{2}\right), \\
& H_{h} \preceq B_{\alpha, p}, \text { for } h(r)=(\log 1 / r)^{1-q}, \quad 1<p<q<\infty \text {. } \\
& B_{\alpha, p} \preceq H_{h}, \quad \text { for } h(r)=r^{n-\alpha p} \quad\left(0 \leqq r \leqq r_{0}<1\right) \text {, } \\
& H_{h} \preceq B_{\alpha, p}, \text { for } h(r)=r^{n-\alpha p}(\log 1 / r)^{1-q}, \quad q>p \text {. }
\end{aligned}
$$

In Theorem 1 and parts (i)(a), (ii)(a) and (b) of Theorem 2, the relation $\preceq$ can be replaced by an inequality of the type: $B^{\prime}(A)$ $\leqq Q B(A)$, for all $A \subset R^{n}, Q$ a constant independent of $A$. Inequalities of this type imply: $B^{\prime}(A)=+\infty$ implies $B(A)=+\infty$. In part (i) (b) of Theorem 2, the $Q$ may depend on the diameter of $A$. In Theorem 3, 
$H_{h}<\infty$ is sufficient for $B=0$.

3. The two principal ingredients that go into proving Theorem 1 in the case wei $B=$ wei $B^{\prime}<n$ are possibly of interest in themselves.

TheOREM 4. Let $k_{1}$ and $k_{2}$ be kernels and $\mu$ a positive Radon measure. Define $u(x)=k_{1} *\left(k_{2} * \mu\right)^{1 /(p-1)}(x)$; then, for all $x$,

$$
\begin{array}{ll}
u(x) \leqq Q^{1 /(p-1)} \sup _{\nu \in \text { Supp } \mu} u(y), & 1<p<2, \\
u(x) \leqq Q \sup _{y \in \text { Supp } \mu} u(y), & 2 \leqq p<\infty .
\end{array}
$$

Here $Q$ is a constant depending only on $n$, the dimension of $R^{n}$. Furthermore, in all cases $u(x) \leqq \sup _{y \in \operatorname{co}(\operatorname{Supp} \mu)} u(y)$.

THEOREM 5. Let $f=f(x)$ be a nonnegative, extended real-valued measurable function on $R^{n}$, then

$$
\left\|g_{\alpha \tau} * f^{t}\right\|_{r} \leqq Q\|f\|_{p}^{t-\tau}\left\|g_{\alpha} * f\right\|_{\alpha}^{r},
$$

with $Q$ a constant independent of $f$ and $0<p<\infty, 1 \leqq q \leqq \infty, 0<\tau<1$, $0<t-\tau<p(1-\tau)$, and $1 / r=(t-\tau) / p+\tau / q$. Here $\|\cdot\|_{p}$ denotes the usual norm in $L_{p}$.

Details of the above theorems will appear elsewhere along with construction of the Cantor sets needed to get the relation $\prec$ in Theorem 1.

\section{REFERENCES}

1. N. Aronszajn and K. T. Smith, Functional spaces and functional completion, Ann. Inst. Fourier Grenoble 6 (1955/56), 125-185. MR 18, 319.

2. L. Carleson, Selected problems on exceptional sets, Van Nostrand Math. Studies, no. 13, Van Nostrand, Princeton, N. J., 1967. MR 37 \#1576.

3. B. Fuglede, On generalized potentials of functions in the Lebesgue classes, Math. Scand. 8 (1960), 287-304. MR 28 \#2241.

4. N. G. Meyers, $A$ theory of capacities for potentials of functions in Lebesgue classes, Math. Scand. 26 (1970), 255-292.

5. J. Serrin, Local behaviour of solutions of quasi-linear equations, Acta Math. 111 (1964), 247-302. MR $30 \# 337$.

6. S. Taylor, On the connexion between Hausdorff measures and generalized capacity, Proc. Cambridge Philos. Soc. 57 (1961), 524-531. MR 24 \#A3254.

University of Minnesota, Minneapolis, Minnesota 55455

Current address: (David R. Adams) Rice University, Houston, Texas 77001 